\title{
Homicídios na região das Américas: magnitude, distribuição e tendências, 1999-2009
}

\author{
Homicides in the Americas region: magnitude, \\ distribution and trends, 1999-2009
}

\author{
Vilma Pinheiro Gawryszewski ${ }^{1}$ \\ Antonio Sanhueza ${ }^{1}$ \\ Ramon Martinez-Piedra ${ }^{1}$ \\ José Antonio Escamilla ${ }^{1}$ \\ Maria de Fátima Marinho de Souza ${ }^{1}$
}

${ }^{1}$ Pan American Health Organization. 525 Twenty-third Street, N.W 20037 Washington DC

USA.gawryszv@paho.org

\begin{abstract}
The scope of this study was to describe the magnitude and distribution of deaths by homicide in the Americas and to analyze the prevailing trends. Deaths by homicide (X85 to Y09 and Y35) were analyzed in 32 countries of the Americas Region from 1999 to 2009, recorded in the Mortality Information System/Pan American Health Organization. A negative binomial model was used to study the trends. There were around 121,297 homicides (89\% men and 11\% women) in the Americas, annually, predominantly in the 15 to 24 and 25 to 39 year age brackets. In 2009 the homicide age-adjusted mortality rate was 15.5/ 100,000 in the region. Countries with lower rates/ 100,000 were Canada (1.8), Argentina (4.4), Cuba (4.8), Chile (5.2), and the United States (5.8), whereas the highest rates $/ 100,000$ were in El Salvador (62.9), Guatemala (51.2), Colombia (42.5), Venezuela (33.2), and Puerto Rico (25.8). From 1999-2009, the homicide trend in the region was stable. They increased in nine countries: Venezuela $(p<0.001)$, Panama $(p<0.001)$, El Salvador $(p<0.001)$, Puerto Rico $(p<0.001)$; decreased in four countries, particularly in Colombia $(p<0.001)$; and were stable in Brazil, the United States, Ecuador and Chile. The increase in Mexico occurred in recent years. Despite all efforts, various countries have high homicide rates and they are on the increase.

Key words External causes, Violence, Homicides
\end{abstract}

Resumo O objetivo do estudo foi descrever a magnitude e a distribuição da mortalidade por homicídios nas Américas e analisar suas tendências. Foram analisados óbitos por homicídios (X85 a Y09e Y35) de 32 países das Américas, período 19992009, registrados no Sistema de Informações de Mortalidade/Organização Pan Americana da Saúde. Utilizou-se modelo binomial negativo para estudar as tendências. Cerca de 121.297 mortes por homicídios ( $89 \%$ homens e $11 \%$ mulheres) ocorreram anualmente nas Américas, predominando as idades de 15 a 24 e de 25 a 39 anos. Em 2009, a taxa padronizada de homicídios da região foi 15,5/ 100.000. Os países com taxas/100.000 baixas foram Canadá (1,8), Argentina (4,4), Cuba (4,8), Chile (5,2) e Estados Unidos (5,8); e com taxas/ 100.000 altas foram El Salvador (62,9), Guatemala (51,2), Colômbia (42,5), Venezuela $(33,2)$ e Porto Rico (25,8). Entre 1999-2009 as taxas da região permaneceram estáveis; aumentaram em nove países, como Venezuela $(p<0,001)$, Panamá ( $p<$ $0,001)$, El Salvador $(p<0,001)$ e Porto Rico ( $p<$ $0,001)$; diminuíram em quatro países, especialmente na Colômbia $(p<0,001)$; e permaneceram estáveis no Brasil, Estados Unidos, Equador e Chile. O aumento no México ocorreu no período mais recente. Apesar dos esforços empreendidos, diversos países têm taxas altas de homicídios e crescimento nas mesmas.

Palavras-chave Causas externas, Violência, Homicídios 


\section{Introdução}

As causas externas, juntamente com as doenças crônico-degenerativas, caracterizam o atual perfil de morbi-mortalidade de muitos países em todo o mundo ${ }^{1}$. Particularmente, a violência está presente na vida de muitas pessoas, em todas as idades, tocando a todos de alguma maneira ${ }^{2}$. Para se construir uma sociedade com menos violência, é essencial conhecer a sua magnitude e características. De forma crescente, a violência vem sendo reconhecida como um problema de sáude pública, dada a magnitude das mortes, internações, demanda em serviços de emergência, reabilitação, além dos impactos gerados na sociedade e, em especial, nas famílias. Relatório publicado pela Organização Mundial da Saúde (OMS) mostrou que a violência encontra-se entre as 10 principais causas de morte e DALY (Disability Adjusted Life Years), globalmente ${ }^{2}$.

Entre as várias formas de expressão da violência, o homicídio pode ser considerado a sua face mais severa. Particularmente a região das Américas tem sido fortemente afetada por este problema, uma vez que as taxas de países como Colômbia, El Salvador, Brasil, Porto Rico, Venezuela e México se encontravam entre as maiores do mundo, de acordo com estimatimativas da OMS para anos próximos a $1999^{2}$. Na verdade, esta situação é mais antiga, uma vez que os estudos realizados na região já apontavam taxas de homicídios altas no Brasil e México, desde a década de 80; e na Colômbia no início dos anos $90^{3-7}$. Certamente que a experiência de altas taxas de homicídio por longos períodos determinam forte impacto na qualidade de vida dessas populações.

Além disso, os homicídios atingem principalmente os jovens, o que se constitui em um obstáculo ao pleno desenvolvimento dos países. Dados do Observatório Regional de Saúde da Organização Pan Americana da Saúde (OPAS) mostram que os homicídios foram a primeira causa de morte entre os indivíduos de sexo masculino de 15 a 29 anos e a segunda causa no sexo feminino na mesma faixa etária ${ }^{8}$. Tais mortes devem ser consideradas socialmente inaceitáveis. Desse modo, analisar os dados acerca dos homicídios na região das Américas é uma tarefa que se faz necessária para a compreensão da situação de violência que afeta vários países. $\mathrm{O}$ ideal é poder contar com dados oportunos para possibilitar uma visão do panorama atual da situação na região.

O presente estudo tem como objetivo descrever a magnitude da mortalidade por homicídios na região das Américas, sua distribuição geográ- fica e analisar as suas tendências, utilizando os dados mais recentes disponíveis.

\section{Método}

\section{Fontes de dados e definição de caso}

A fonte de dados utilizada para este estudo foi o Sistema de Informações de Mortalidade da OPAS/ OMS, que reúne os bancos de dados disponibilizados anualmente pelos países da Região das Américas, através dos Ministérios de Saúde ou Institutos de Estatísticas. Esse banco contém dados provenientes das declarações de óbito e são as fontes oficiais de informação de mortalidade dos países. O Sistema é gerenciado pela área de Informação e Análise de Saúde/OPAS que avalia a integridade e a uniformidade dos dados enviados, além de realizar a validação de variáveis selecionadas (sexo, idade e causa básica de morte).

Primeiramente foram selecionados todos os óbitos cuja causa básica de morte estava codificada no Capítulo XX (causas externas) da Classificação Internacional de Doenças (CID-10) ${ }^{9}$. Para as finalidades deste estudo considerou-se como homicídio os óbitos cuja causa básica de morte estava codificada com os códigos de agressões (X85 a Y09) e também aqueles classificados como intervenções legais (Y35). Para apresentar os eventos de intenção indeterminada foram selecionados os óbitos classificados com os códigos de Y10 a Y34. Estes eventos são importantes de serem quantificados, pois permitem verificar possíveis influências da qualidade da informação dos homicídios. Para a descrição da situação atual foi utilizado os dados do último ano disponível, 2009, exceto para Guiana e Trinidade \& Tobago que disponibilizaram dados para 2008. Como ponto inicial para a análise das tendências buscou-se utilizar os dados a partir de 1999, mas houve pequena variação entre os países, dependendo da disponibilidade dos dados.

O banco de dados analisado continha dados provenientes de 32 países. Optou-se por apresentar informações para os 20 países com maior população, uma vez que os países com população e número de mortes pequenos, muitas vezes apresentam flutuações nas taxas de mortalidade. Esse banco contém as mortes informadas pelos países, sem aplicação de correção para subregistro de mortes. Também não foram apresentadas análises de países com baixa qualidade de informação para os homicídios. Entretanto, esses países foram incluídos nos cálculos das ta- 
xas de mortalidade para a Região das Américas. Para o cálculo das taxas foram utilizados dados populacionais provenientes da Divisão de População das Nações Unidas ${ }^{10}$. Para assegurar comparabilidade entre os países as taxas foram padronizadas pelo método direto usando a População Mundial da OMS ${ }^{11}$.

Por se tratar de um estudo que usa dados secundários anônimos, a aprovação de comitê de ética não é requerida.

\section{Análises dos dados}

Foi realizada uma análise descritiva dos dados de mortalidade, incluindo o cálculo de taxas padronizadas e específicas por idade, para o período 1999-2009. Os dados estão apresentados sob a forma de números absolutos, proporções e taxas padronizadas por 100.000 habitantes. Foram construídos gráficos para apresentar as séries temporais. Adicionalmente, as taxas do último ano disponível foram ordenadas em tercis e apresentadas sob a forma de mapa.

Inicialmente, exploramos as séries temporais usando os modelos de regressão linear e binomial negativo. O primeiro usou o logaritmo das taxas de mortalidade e o último usou o número de mortes diretamente. Em razão da variação nas taxas de alguns países, o modelo que melhor se ajustou foi o binomial negativo, que proporcionou os valores-p das tendências e dos riscos relativos associados às variáveis país, sexo e idade. Os resultados do modelo somente serão descritos no texto para os países com menores flutuações nos dados. Todas as análises foram realizadas usando o programa estatístico SAS ${ }^{12}$.

\section{Resultados}

\section{Magnitude e tendência da mortalidade nas Américas}

No período do estudo, 1999 a 2009, a Região das Américas registrou em média cerca de 5,2 milhões de óbitos anuais, das quais 497 mil (cerca de $10 \%$ do total) foram devidos a causas externas. Entre estas mortes, 37\% eram decorrentes de violências ou causas intencionais, 56\% eram não intencionais e 7\% foram eventos com intenção indeterminada.

Nesse período, o total de homicídios (X85Y09, Y35, CIE-10) na região foi 1.334.271, dos quais 1.191.905 eram homens e 142.366 mulheres. A média anual de homicídios foi aproxima- damente 121.297, dos quais cerca de 108.355 eram homens ( $89 \%$ do total) e 12.942 mulheres (11\% do total). A Figura 1 mostra a distribuição do número de mortes por homicídios segundo sexo e faixa etária, para todo o período de estudo, sendo possível observar a predominância do sexo masculino em todas as faixas etárias e também que as faixas de 15 a 24 e 25 a 39 anos foram aquelas que contribuiram com o maior número de mortes para o total.

A taxa padronizada de mortalidade por homicídios nas Américas em 2009 foi 15,5/100.000 (27,8 para os homens e 3,2 para as mulheres). Observou-se uma tendência de estabilidade nas taxas, sendo que a variação nas taxas de 1999 versus 2009 foi de 2,8\%. Entretanto, a observação da curva mostrou um declínio leve nas taxas até 2007 quando passam a aumentar. A Figura 2 mostra os riscos de morrer por homicídios para o período 1999-2009 onde é possível observar diferenças importantes entre as faixas etárias. Os menores de 15 anos tiveram as taxas mais baixas, variando entre 1,4 e 1,6 por 100.000 habitantes. A partir dos 15 anos são observados aumentos importantes no risco com taxas variando entre 27,2 e 32,4 por 100.000 habitantes no grupo de 15 a 24 anos; e de 24,6 e 28,5 por 100.000 no grupo de 25 a 39 anos. A partir dos 40 anos as taxas diminuem, mantendo-se entre 11,3 e 13,5

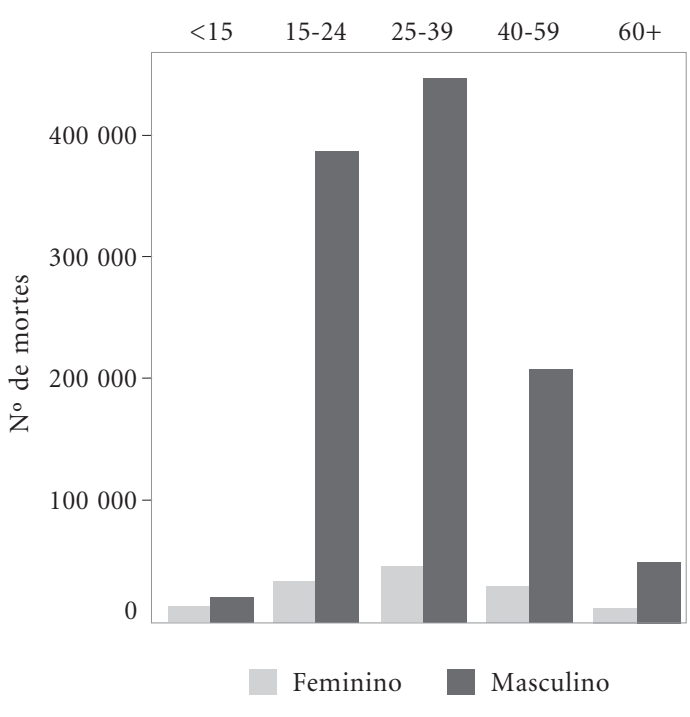

Figura 1. Mortalidade homicídios (X85-Y09 + Y35) (número de mortes) segundo sexo e faixa etária. Região das Américas , 1999-2009. 


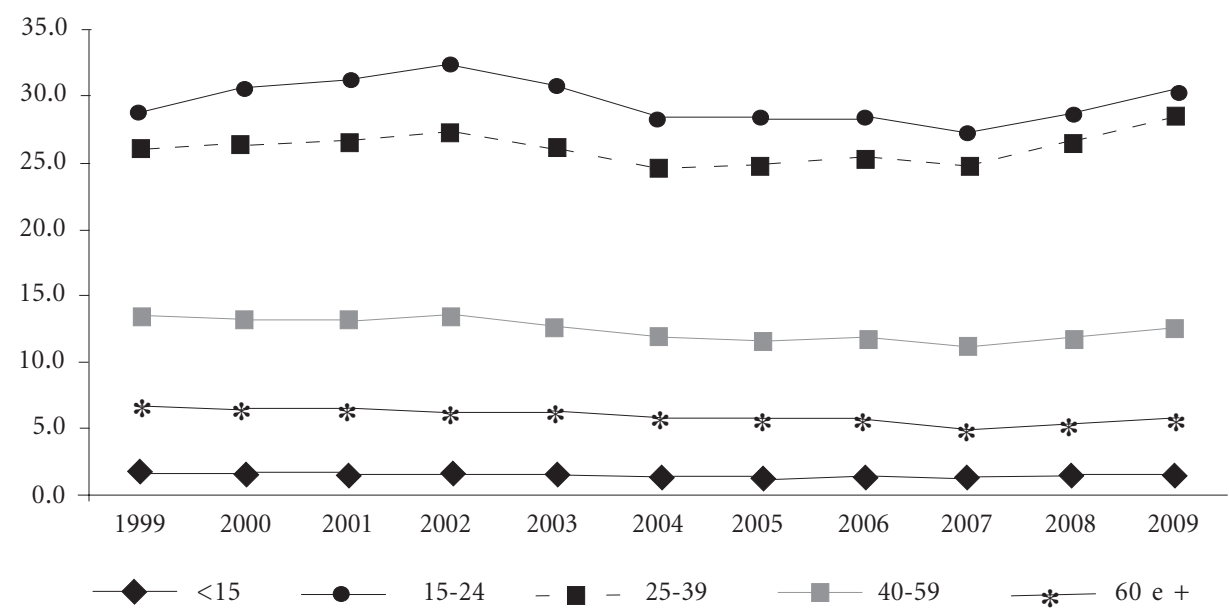

Figura 2. Tendência da mortalidade por homicídios (X85-Y09 + Y35) (taxa padronizada por 100.000 habitantes) segundo faixa etária. Região das Américas, 1999-2009.

por 100.000 , para o grupo de 40 a 59 anos; e de 5,1 a 6,7 por 100.000 para os de 60 anos e mais. Embora os diferenciais por idade sejam importantes, as tendências da mortalidade para as Américas mostraram relativa estabilidade entre 1999 e 2006. No entanto, a partir de 2007 um aumento é registrado nas taxas, em particular o grupo de 15 a 39 anos. Usando os dados de todo o período, ao comparar o grupo de menor risco, menores de 15 anos, com os outros grupos de idade foi observado que o risco de morrer para os 15 a 24 anos foi quase 20 vezes maior $(\mathrm{RR}=$ 19,3; [ IC 95\% 18,9-19,8]) e os de 25 a 39 anos 17 vezes $(\mathrm{RR}=17,1 ;[$ IC $95 \% 16,7-17,5])$.

\section{Magnitude da mortalidade por homicídios nos países}

A Tabela 1 mostra a magnitude da mortalidade por homicídios nos países da região, para o último ano com informação disponível, através do número de mortes, taxas padronizadas por 100.000 habitantes por sexo e proporção dos homicídios no total de mortes por causas externas. Adicionalmente, a distribuição espacial das taxas de homicídios é apresentada na Figura 3. Os países que apresentaram as taxas mais baixas (de 1 a 9) foram Costa Rica, Estados Unidos, Chile, Cuba, Argentina e Canadá. Entre os países considerados como taxas médias (de 10 a 24) se encontram Panamá, México, Equador, Paraguai,
Suriname e Nicarágua. É possível observar que os países classificados como taxas altas (de 25 a 63) foram El Salvador, Guatemala, Colômbia, Venezuela, Porto Rico e Brasil. A sobremortalidade masculina foi observada em todos os países. Ao observar a razão masculino/feminino verificou-se que Venezuela, Porto Rico, Colômbia, Brasil, Equador e Nicarágua foram os países que apresentaram valores mais elevados $(17,1$ a 10,1) enquanto que Costa Rica, Argentina, Suriname, Estados Unidos, Canadá e Cuba apresentaram os valores mais baixos.

Também é possível observar na Tabela 1 que a proporção dos homicídios no total das causas externas, variou de 3,7\% no Canadá a 59,1\% em El Salvador. Com respeito à proporção de óbitos decorrentes de eventos de intenção não determinada em relação ao total de óbitos por causas externas, os países com proporções mais altas (de $7,1 \%$ a $14,6 \%$ ) foram Argentina, Venezuela, Guiana, Brasil, Equador e Guatemala; e com proporções intermediárias (de 4,2\% a 6,7\%) foram Panamá, Suriname, Colômbia, Trinidade \& Tobago, Nicarágua, Canadá e México. Proporções baixas, menores que $4,2 \%$, foram observadas em Costa Rica, Cuba, Paraguai, Estados Unidos, Porto Rico, El Salvador e Chile. É importante considerar que entre estas mortes, podem estar incluídos alguns casos de homicídios, contribuindo assim para a subestimação das taxas de homicídios. 
1. Homicídios (X85-Y09 + Y35) e eventos de intenção não determinada (Y10-Y34) segundo sexo (mortes, taxas padronizadas/100.000 habitantes). Países selecionados das Américas, último ano disponível.

\begin{tabular}{|c|c|c|c|c|c|c|c|c|c|}
\hline \multirow[b]{2}{*}{ País } & \multirow[b]{2}{*}{ Ano } & \multicolumn{6}{|c|}{ Homicidíos } & \multicolumn{2}{|c|}{$\begin{array}{c}\text { Intenção } \\
\text { indeterminada }\end{array}$} \\
\hline & & $\begin{array}{c}\text { Mortes } \\
\mathrm{N}\end{array}$ & $\begin{array}{c}\text { Taxa/ } \\
100.000 \\
\text { Total }\end{array}$ & $\begin{array}{c}\text { Taxa/ } \\
100.000 \\
\text { Masc. }\end{array}$ & $\begin{array}{c}\text { Taxa/ } \\
100.000 \\
\text { Fem. }\end{array}$ & $\begin{array}{c}\text { Razão } \\
\text { M/F }\end{array}$ & $\begin{array}{c}\% \mathrm{CE}^{\star} \\
\text { Total }\end{array}$ & $\begin{array}{l}\text { Mortes } \\
\mathrm{N}\end{array}$ & $\begin{array}{c}\% \\
\mathrm{CE}^{\star *} \\
\text { Total } \\
\end{array}$ \\
\hline Argentina & 2009 & 1760 & 4.4 & 7.6 & 1.2 & 4.4 & 9.7 & 2659 & 14.6 \\
\hline Brazil & 2009 & 50907 & 25.2 & 46.8 & 4.2 & 25.2 & 37.3 & 12738 & 9.3 \\
\hline Canada & 2009 & 581 & 1.8 & 2.7 & 0.9 & 1.8 & 3.7 & 685 & 4.4 \\
\hline Chile & 2009 & 903 & 5.2 & 9.3 & 1.1 & 5.2 & 11.1 & 1 & 0.0 \\
\hline Colombia & 2009 & 19541 & 42.5 & 79.4 & 7.0 & 42.5 & 56.1 & 2116 & 6.1 \\
\hline Costa Rica & 2009 & 408 & 8.7 & 15.2 & 1.9 & 8.7 & 20.7 & 79 & 4.0 \\
\hline Cuba & 2009 & 564 & 4.8 & 6.9 & 2.6 & 4.8 & 7.9 & 225 & 3.1 \\
\hline Ecuador & 2009 & 2169 & 15.6 & 28.6 & 2.7 & 15.6 & 22.6 & 681 & 7.1 \\
\hline El Salvador & 2009 & 3763 & 62.9 & 120.2 & 14.6 & 62.9 & 59.1 & 1 & 0.0 \\
\hline Guatemala & 2009 & 5956 & 51.2 & 96.6 & 11.3 & 51.2 & 46.8 & 897 & 7.1 \\
\hline Guyana & 2008 & 118 & 17.9 & 28.4 & 7.4 & 17.9 & 18.5 & 60 & 9.4 \\
\hline Mexico & 2009 & 18461 & 16.8 & 31.0 & 3.2 & 16.8 & 29.1 & 2638 & 4.2 \\
\hline Nicaragua & 2009 & 495 & 9.4 & 17.4 & 1.7 & 9.4 & 21.6 & 126 & 5.5 \\
\hline Panama & 2009 & 786 & 22.7 & 40.9 & 4.1 & 22.7 & 40.2 & 131 & 6.7 \\
\hline Paraguay & 2009 & 648 & 11.3 & 20.0 & 2.4 & 11.3 & 21.5 & 93 & 3.1 \\
\hline Puerto Rico & 2009 & 922 & 25.8 & 49.4 & 2.9 & 25.8 & 40.2 & 7 & 0.3 \\
\hline Suriname & 2009 & 35 & 7.1 & 12.0 & 2.7 & 7.1 & 8.5 & 26 & 6.3 \\
\hline Trinidad \& Tobago & 2008 & 594 & 39.7 & 73.3 & 7.6 & 39.7 & 43.4 & 81 & 5.9 \\
\hline United States & 2009 & 17045 & 5.8 & 9.1 & 2.4 & 5.8 & 9.5 & 5012 & 2.8 \\
\hline Venezuela & 2009 & 9772 & 33.2 & 62.4 & 3.7 & 33.2 & 33.3 & 4025 & 13.7 \\
\hline
\end{tabular}

" Proporcão de homicídios no total das causas externas; ${ }^{* *}$ Proporcão de eventos de intenção indeterminada no total das causas externas

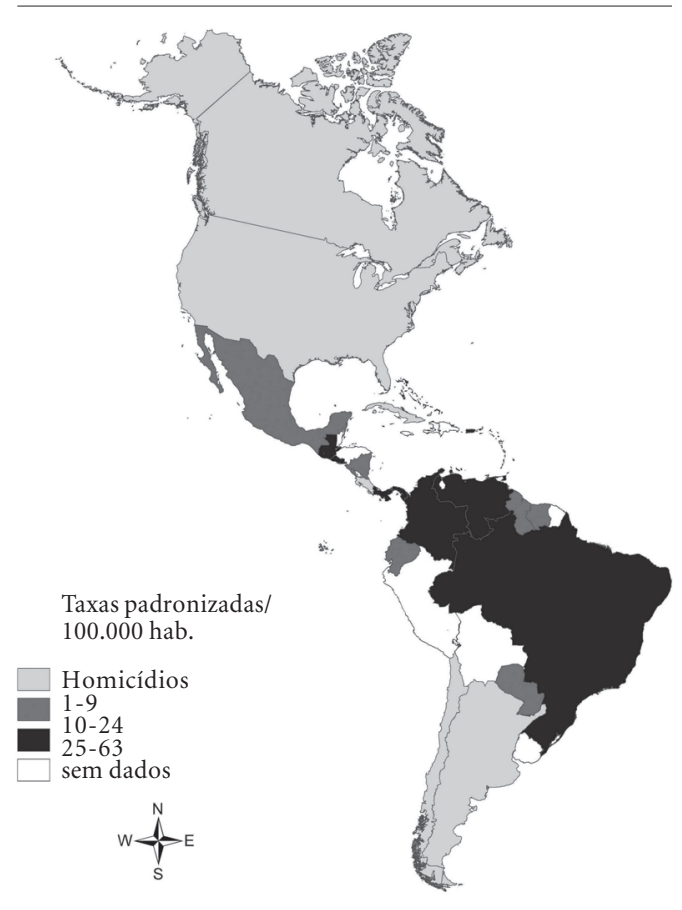

Figura 3. Mortalidade por homicídios (X85-Y09+ Y35) (taxas padronizadas/ 100.000 habitantes). Região das Américas, 2009
Usando os dados para todo período de estudo, o cálculo do risco relativo dos países a partir do modelo binomial negativo mostrou que, a probalidade de morte por homicídio na Colômbia foi 34,3 vezes (IC 95\% 18,9-19,8) a do Canadá (usado como referência, por apresentar as menores taxas no período), em El Salvador foi 28,1 vezes (IC 95\% 24,0-33,0), na Guatemal 20,8 (IC 95\% 17,0-25,4), na Venezuela 19.4 vezes (IC 95\% 16,6-22,8), no Brasil 16,4 vezes (IC 95\% 14,0-19,3), e em Trinidad e Tobago 15,9 vezes (IC 95\% 13,4-18,9). Probabilidades menores de morte, na comparação com o Canadá, foram encontradas no Chile 3,2 vezes (IC 95\% 2,7-3,7), Cuba 3,4 vezes (IC 95\% 2,9-4,0), Estados Unidos 3,8 vezes (IC 95\% 3,3,0-4,5), e Costa Rica 3,8 vezes (IC 95\% 3,2-4,5).

\section{Tendências da mortalidade nos países}

As Figuras 4a, 4b e 4c apresentam as tendências das taxas padronizadas de homicídios nos diferentes países segundo sexo. Para a análise das tendências foram examinadas a curva de cada 
Países com taxas altas (25-63/100.000 hab.)
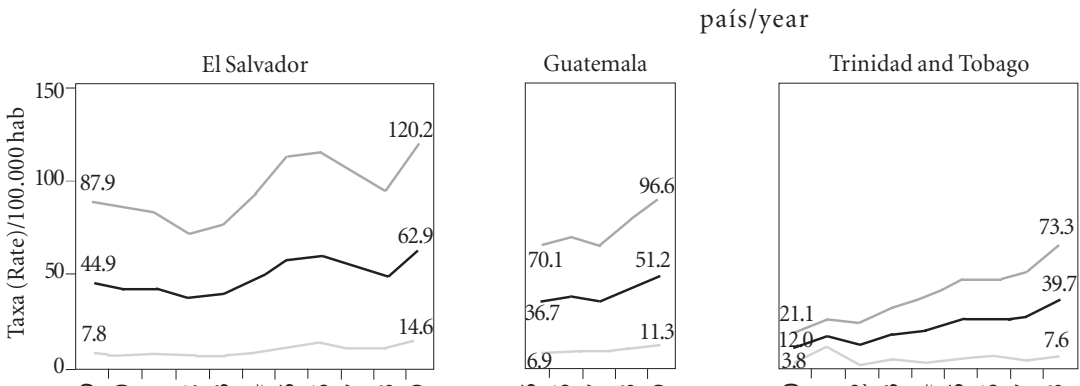

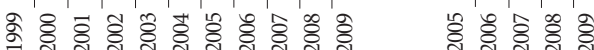
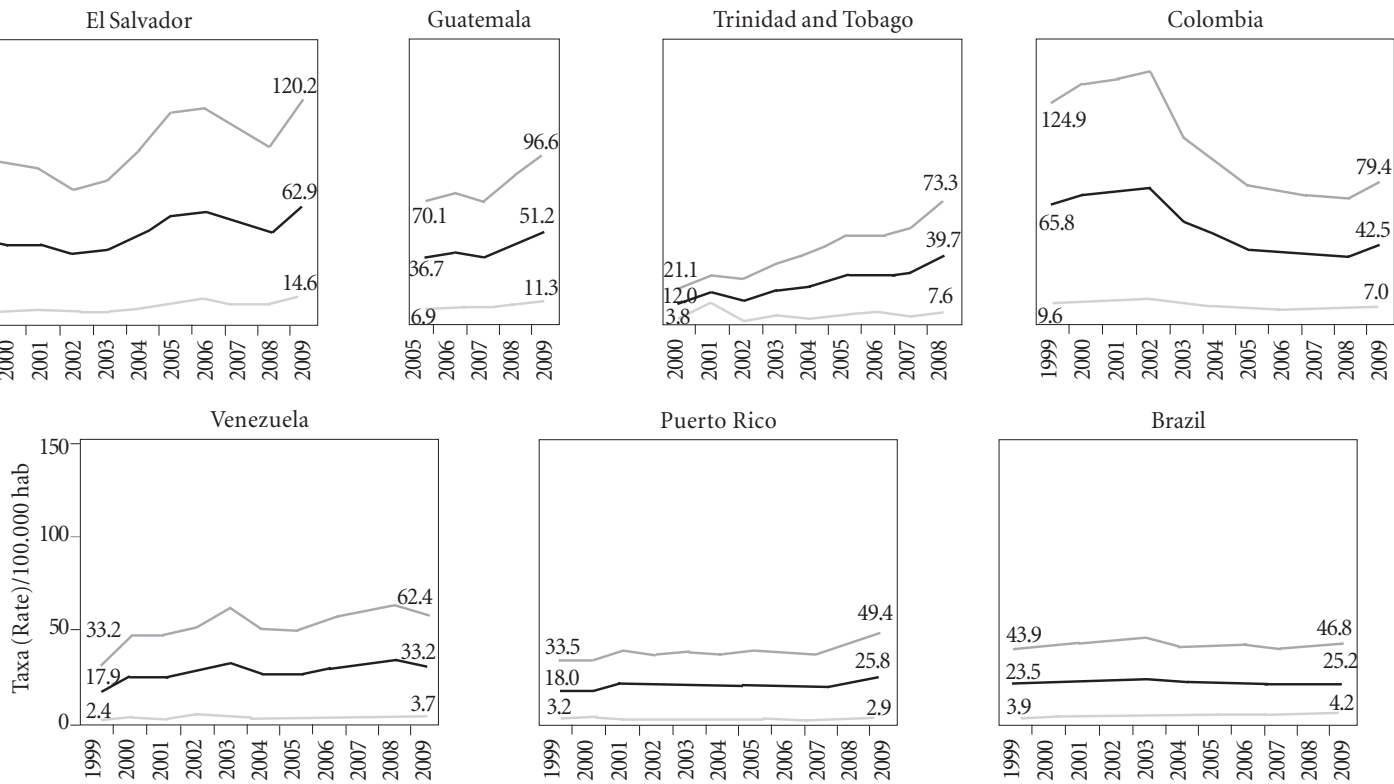

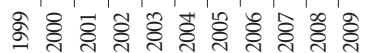

Masculino

Total

Feminino

Figura 4a. Tendências dos homicídios (X85-Y09 + Y35) (taxas padronizadas/100.000 habitantes) segundo país. Região das Américas, 1999-2009.

Países com taxas intermediárias (10-24/100.000 hab.)

país/year
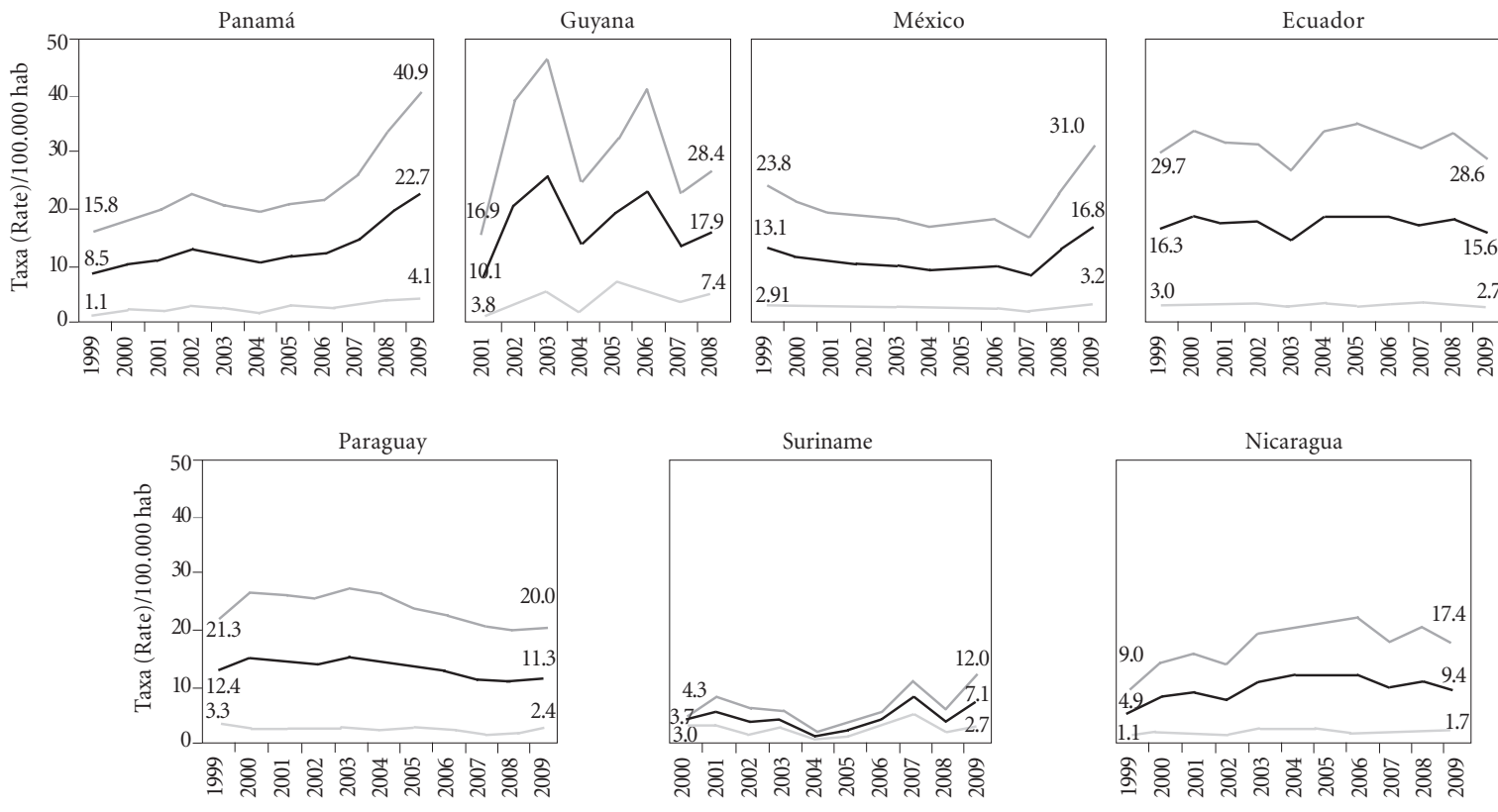

Masculino

Total

Feminino

Figura 4b. Tendências dos homicídios (X85-Y09 + Y35) (taxas padronizadas/100.000 habitantes) segundo país. Região das Américas, 1999-2009. 

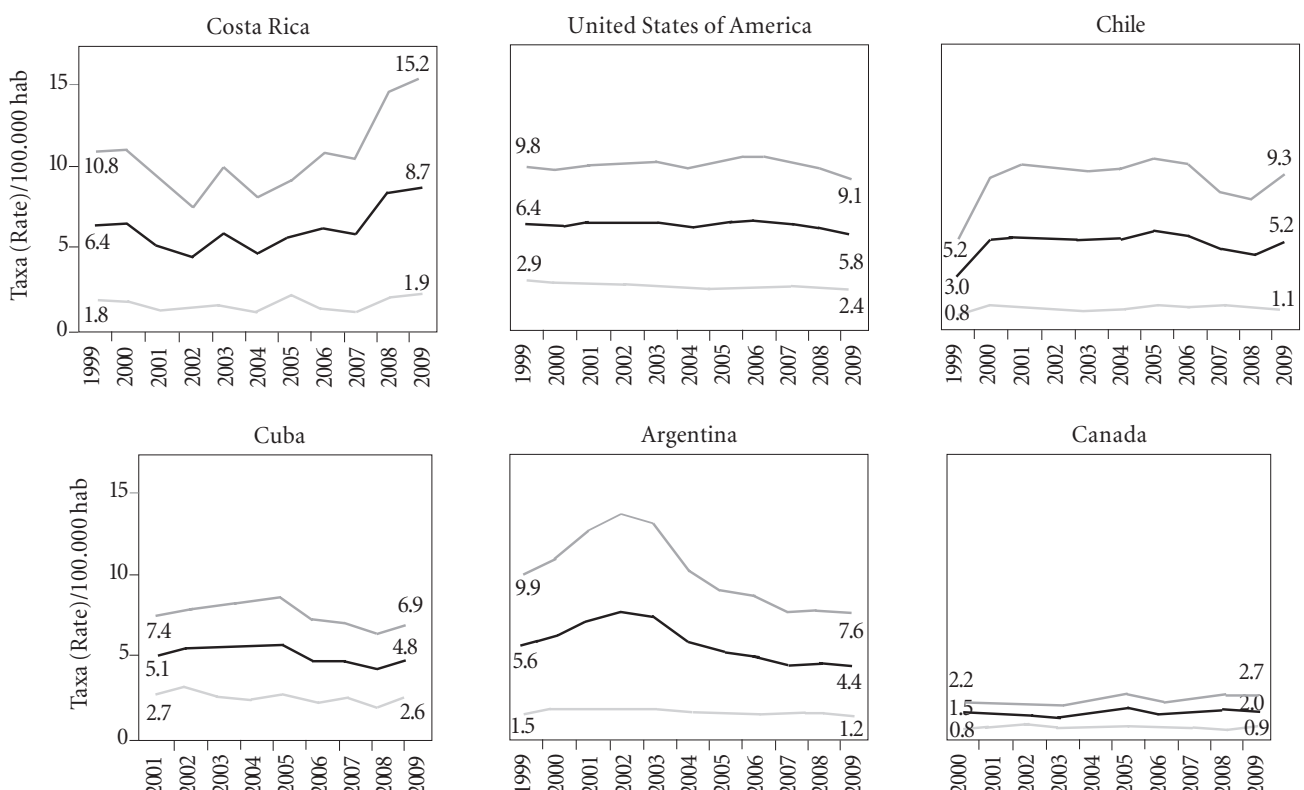

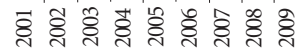

Masculino

Total

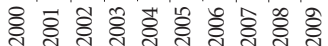

Figura 4c. Tendências dos homicídios (X85-Y09 + Y35) (taxas padronizadas/100.000 habitantes) segundo país. Região das Américas, 1999-2009.

país, as taxas ano a ano, a diferença percentual entre as taxas no início versus fim do período, além dos resultados do modelo binomial negativo. No período de estudo, um total de nove países apresentaram tendência de aumento em suas taxas, em quatro países a tendência foi de descenso, em outros seis países houve tendência de estabilidade e considerou-se o México como um caso particular.

Os países classificados no grupo de taxas baixas $(1-9 / 100,000)$ já iniciaram o período de estudo (1999 ou 2000) com taxas baixas. Observouse tendência de declínio em dois países. A Argentina apresentou um pequeno aumento em suas taxas de 2000 a 2003, quando então passam a descrescer; e no período total (1999 versus 2009) acumulou uma redução de $-22 \%$ com variação anual média estatisticamente significativa $(\mathrm{p}<$ $0,001)$. Cuba mostrou um leve descenso na mortalidade por homicídios (-7\%) entre 2000 e 2009 , com variação anual média estatisticamente significativa $(\mathrm{p}<0.01)$. Dois países apresentaram tendência de estabilidade, uma vez que o aumento verificado no Chile e o leve decréscimo nos
Estados Unidos não foram estatisticamente significativos. Por outro lado, a tendência do Canadá foi de leve crescimento, cerca de $18 \%$ no período de estudo, com variação anual média estatisticamente significativa ( $\mathrm{p}<0,05)$, mas ressalta-se que esse país segue detendo as taxas mais baixas da região. A Costa Rica acumulou um aumento de $22 \%$ no período estudado, sendo que a variação anual média foi estatisticamente significativa $(\mathrm{p}<0.01)$; porém esses resultados devem ser vistos com cuidado, uma vez que as taxas se apresentaram instáveis no período.

Os países com taxas padronizadas de homicídios classificadas como intermediárias, entre 10 e 24/100.000 iniciam a série histórica com suas taxas entre esses valores. Nesse grupo, três países apresentaram uma tendência de crescimento no período de 1999 a 2009. O maior aumento foi observado no Panamá (138\%), cuja variação média anual foi estatisticamente significativa ( $\mathrm{p}$ $<0,001)$, destacando-se o período a partir de 2007. Também a Nicarágua mostrou um incremento de 39\% nas taxas no período, com variação anual média estatisticamente significativa ( $\mathrm{p}$ 
$<0,01)$. A tendência no México mostrou um comportamento particular, com um declínio de $-37 \%$ de 1999 a 2007, quando então passam a aumentar marcadamente, cerca de $103 \%$ entre 2007 e 2009. A variação anual média calculada para o período total não foi estatisticamente significativa, porém foi significativa considerando o período 2007 a 2009. A mortalidade por homicídios no Equador apresentou-se estável, uma vez que o leve decréscimo observado nas taxas não se mostrou estatisticamente significativo. Pela mesma razão, as tendências da Guyana e do Suriname foram consideradas de estabilidade, porém deve ser considerada a forte instabilidade observada nas taxas no período na Guyana e o número pequeno de mortes no Suriname. No Paraguai observou-se uma diminuição leve das taxas (-9\%), com variação anual média estatisticamente significativa $(\mathrm{p}<0,05)$.

O comportamento das tendências, entre 1999 e 2009, nos países com taxas de homicídios altas, entre 25 e 63/100.000, foi de aumento na maioria dos países, exceto Colombia e Brasil. As taxas da Colômbia encontravam-se em ascensão até 2002, quando então passam a declinar significativamente, $44 \%$ entre 2002 e 2009 , sendo que a variação anual média calculada para todo o período foi estatisticamente significativa ( $p<0,001)$. A tendência do Brasil se mostrou estável, uma vez que a variação anual média não foi estatiticamente significativa. Assinala-se que a curva do Brasil mostra um decréscimo leve nas taxas entre 2004 e 2007 e discreto aumento entre 2007 e 2009 . Por outro lado, no período de estudo foram observados incrementos de $40 \%$ nas taxas em El Salvador, $86 \%$ em Venezuela e de $41 \%$ em Porto Rico. Nestes três países a variação anual média foi estatisticamente significativa $(\mathrm{p}<0,001)$. Também a Guatemala, entre 2005 e 2009, teve aumento em suas taxas de $40 \%$, com variação anual média estatisticamente significativa $(\mathrm{p}<0,001)$.

As tendências por sexo acompanharam a da população total, especialmente as masculinas. No entanto, cabe chamar atenção para o aumento dos homicídios entre os homens na Costa Rica, especialmente a partir de 2008, que não foi acompanhado pelo mesmo aumento entre as mulheres. Também em Porto Rico, verificou-se um aumento das taxas masculinas no final do período estudado, enquanto que as taxas femininas se mantiveram estáveis.

\section{Discussão}

Os nossos achados evidenciaram que, apesar dos esforços que vêm sendo realizados por vários países, a mortalidade por homicídios permanece como um grande problema nas Américas, uma vez que diversos países exibem taxas altas. Além disso, a avaliação das tendências mostrou que embora as taxas da região se apresentem estáveis, vários países incluídos neste estudo tiveram um crescimento na última década. Na realidade, tais incrementos foram observados especialmente entre aqueles países com taxas médias e altas. Sem dúvida, as taxas da região estão fortemente influenciadas pelas taxas dos países com maiores populações, tais como os Estados Unidos, Brasil e México, que respondem por $66 \%$ da população total da região (estimativas de 2011) ${ }^{13}$. De acordo com nossos achados, nos dois primeiros países a tendência encontrada foi de estabilidade e no México o crescimento nas taxas se deu mais recentemente.

Entre os países que obtiveram avanços na redução da mortalidade por homicídios, destaca-se a Colômbia que, embora ainda permaneça com taxas altas, não ocupa mais o primeiro lugar entre os países, quando se compara os valores atuais com os do início da série e também com os divulgados em informe da $\mathrm{OMS}^{2}$. Estudos realizados nesse país apontam que houve um longo período de altas taxas de homicídio, com elevações importantes de 1991-1993 e de 2000$2002^{6}$. O número de homens mortos no país por homicídio, cerca de 490 mil, no período 1985 a 2006, representou $21 \%$ do total de mortes neste sexo ${ }^{6}$. Possivelmente políticas governamentais implementadas e adoções de medidas de prevenção em algumas cidades levaram ao seu descenso, a partir de então. Por exemplo, em Medellín, Colômbia, foi verificado maiores declínios nas taxas de homicídios em áreas de baixa renda onde foram realizados investimentos e intervenções na infraestrutura, comparativamente às áreas controle ${ }^{14}$

Também deve ser considerado promissor a tendência de estabilidade observada no Brasil na última década, visto que o país apresentava taxas em crescimento desde os anos 80 . A leve queda na mortalidade por homicídios entre 2004 e 2007 é consistente com a literatura ${ }^{5}$, porém observamos que em 2008 e 2009 as taxas voltaram a aumentar levemente. Neste período, enquanto algumas capitais brasileiras alcançaram reduções expressivas, como São Paulo, outras experimentaram aumento em suas taxas ${ }^{15-16}$. Entre 1991 e 
2007 as taxas de mortalidade por homicídios dimiuíram nas regiões Sudeste, Norte e Centro Oeste mas aumentaram no Nordeste e Sul. ${ }^{5}$ Isto pode indicar um padrão de migração da violência, apontando a necessidade de seguir monitorando o fenômeno no país. Particularmente, a redução dos homicídios no município de São Paulo mostrou correlação com a queda do desemprego, investimentos em políticas sociais (educação, sáude e saneamento) e mudanças nas políticas de segurança pública (armas apreendidas e encarceramento-aprisio-namento) ${ }^{16}$. Outro país com estabilidade nas taxas no último decênio foram os Estados Unidos, cuja queda nos homicídios se deu nos anos $90^{17}$.

Entre os países que apresentaram tendência de crescimento em suas taxas, destaca-se o aumento marcado no México, a partir de 2008. Além disso, estudo anterior mostrava uma tendência de queda nos homicídios entre 1979 a 1992 no país ${ }^{7}$. Este recente incremento coincidiu com o início de uma estratégia do governo de luta frontal contra o crime organizado em $2007^{18}$. Perversamente, somente entre 2007 e 2010 os homicídios em menores de 15 anos cresceram 100\% ${ }^{19}$. Dados da Venezuela demonstram que o crescimento dos homicídios já vem acontecendo há mais tempo, uma vez que as taxas duplicaram entre 1989 e $1999^{20}$; também nossos resultados mostraram duplicação das taxas no último decênio. O Panamá experimentou aumento em suas taxas no período do estudo e também anteriormente, quando as taxas quadruplicaram entre 1980 a 1988, especialmente cerca de 1989, ano da ocorrência de um conflito bélico no país ${ }^{4}$. Também estudos anteriores apontaram que as taxas de El Salvador estiveram entre as mais altas da região ${ }^{4}$ e globalmente ${ }^{1}$. Em relação à Costa Rica, nossos achados mostram um incremento expressivo das taxas em 2008, o que é consistente com estudo conduzido no próprio país ${ }^{21}$.

Homicídio é um tema de grande complexidade, uma vez que muitos fatores, individuais, culturais, sociais e econômicos concorrem para a sua ocorrência. Entre esses fatores, em nossa região, cabe destacar as desigualdades sociais e o narcotráfico. Os nossos achados mostraram que os países com taxas de homicídios mais elevadas na região foram El Salvador, Guatemala, Trinidade \& Tobago e Colômbia. Por outro lado, os países com menores riscos foram o Canadá, Estados Unidos Chile, Cuba, e Argentina. Os países no primeiro grupo apresentam indicadores socioeconômicos e demográficos mais desfavoráveis que os do segundo grupo ${ }^{13}$. Embora em nosso estudo não tenham sido incluídos dados da Jamaica, análise realizada com dados provenientes de registos policiais nesse país mostrou que as taxas de homicídios na população masculina de 15-44 anos, nos anos de 1998-2002 chegou a 121/ $100,000^{22}$; esse valor é um pouco maior que a taxa do Brasil para a mesma faixa e período.

A relação do narcotráfico com os homicídios é uma questão de difícil abordagem pela área da saúde, por isso a importância do trabalho interdisciplinar neste tema. Reichenheim et al. citaram estudos que evidenciaram associação entre homicídios, narcotráfico e posse ilegal de armas no Brasil ${ }^{5}$. Considerou-se que o aumento nas taxas de homicídios na Colômbia nos anos de 19911993 foi consequencia do incremento no tráfico de drogas ${ }^{6}$. No México foi observado que o narcotráfico teve um peso maior na variação de taxas de homicídios de adolescentes e de jovens entre os estados ${ }^{23}$. Em Nova Iorque, um dos fatores associados com a redução dos homicídios entre a população negra foi o decréscimo no consumo de cocaína. De acordo com informe da Organização das Nações Unidas ${ }^{24}$, globalmente, o cultivo da cocaína se concentra em Colombia, Perú e Bolívia. As ações governamentais voltadas para o combate ao narcotráfico na Colômbia e México concorreram para que outros países na região, tais como Bolívia, Venezuela, Equador e outros na América Central, passassem a ser utilizados como países de trânsito. Mas, cabe ressaltar que outros fatores devem estar presentes nessa relação, pois este mesmo relatório aponta que a América do Norte é o maior mercado regional consumidor de cocaína ( $40 \%$ globalmente), onde se verifica taxas mais baixas de homicídio comparativamente aos outros países na região.

Franco ${ }^{25}$ introduz o conceito de um contexto explicativo, onde uma combinação de fatores culturais, econômicos, sociopolíticos e legais permitem que o fenômeno da violência seja históricamente possível. Segundo o autor, contribuem para essa situação as chamadas condições estruturais e as condições transitórias. As estruturais são aqueles componentes fundamentais e de longa duração que estão relacionados com o fenômeno da violência. As transitórias, por outro lado, tem curta duração e exercem um complemento importante para os componentes fundamentais. No caso da violência essa diferenciação é útil quando se procura possíveis soluções para o problema.

Os nossos achados acerca da sobremortalidade masculina nos homicídios em todos os países é consistente com a literatura. Por outro lado, considera-se importante examinar a grande va- 
riação encontrada na razão de masculinidade entre os países. Possivelmente, os valores mais baixos indicam um maior peso da violência intrafamiliar na composição dessas mortes, expressadas pelo feminicídio. Tal fato, dada a sua relevância, mereceria a realização de estudos posteriores para melhor elucidar o fenômeno.

Algumas limitações têm que ser levadas em consideração na interpretação desses resultados. A primeira é que as taxas de mortalidade apresentadas podem estar subestimadas em alguns países, por problemas de cobertura/sub-registro de dados de mortalidade. Proporção de subregistro acima de $10 \%$ foi encontrada em Costa Rica, Guatemala, Nicarágua e Paraguai ${ }^{13}$. Também a proporção de mortes classificados como eventos de intenção indeterminada pode ter contribuído para a subestimação das taxas de Venezuela, Argentina, Canadá e Brasil. Tal fato dificulta o real dimensionamento e o monitoramento do problema. Se investigadas, provavelmente, uma parcela dessas mortes seriam classificadas como homicídios. Por exemplo, investigação realizada em Vitória, cidade brasileira com altas taxas de homicídios, possibilitou esclarecer que entre as mortes com intenção indeterminada, $69 \%$ eram homicídios e $20 \%$ suicídios $^{26}$. Uma outra limitação diz respeito ao modelo binomial negativo, que mesmo tendo sido usado para a análise das tendência por seu melhor ajuste ao conjunto de dados, as flutuações ou inflexões acentudas nas taxas de mortalidade em alguns país no período estudado, trouxe dificuldades de interpretação dos resultados do modelo.

Entre os pontos fortes do presente estudo, destaca-se a disponibilização de dados atuais e tendências recentes, além de apresentar informações para alguns países com maior dificuldade para analizar rotineiramente seus dados de homicídios. Os autores esperam contribuir para a discussão do problema e a tomada de decisões para o seu controle e prevenção. A violência deve ser vista como um processo que inclui as condições que permitem que ela aconteça, suas diferentes formas de expressão e suas consequencias individuais e coletivas.

\section{Conclusões}

O estudo dos homicídios na Região das Américas usou os dados provenientes do Sistema de Informações de Mortalidade da OPAS e possibilitou fornecer um panorama da situação atual do problema na Região e nos países, o que pode auxiliar na implantação de ações preventivas. As diferenças encontradas nos diversos países reforçam a necessidade desse monitoramento integrado. É preciso chamar atenção para a preponderância do sexo masculino, e que duas faixas etárias merecem ações específicas, os adolescentes, os jovens e os adultos de 14 a 24 anos e de 25 a 39 anos.

Vários países da região mantêm taxas muito altas de homicídios e em nove dos vinte apresentados verificou-se crescimento. Entretanto, a redução nas taxas de homicídios observada em alguns países indica que existem saídas para o problema da violência. É necessário que a discussão destas medidas e de políticas seja feita com toda a sociedade. Desse modo, recomenda-se o aprofundamento de estudos que possam identificar quais são essas intervenções bem sucedidas e como manter as conquistas. Por fim, espera-se que os resultados deste estudo possam estimular o desenvolvimento de posteriores análises nos países. 


\section{Colaboradores}

Todos os autores participaram do delineamento da análise, interpretação dos dados e aprovação da versão a ser publicada. VP Gawryszewski participou da análise e redação do artigo. A Sanhueza realizou a análise estatística dos dados. $\mathrm{R}$ Martinez-Piedra participou da análise dos dados e revisão do artigo. JA Escamilla participou da análise e revisão do artigo. MF Marinho de Souza da concepção e revisão crítica do artigo.

\section{Referências}

1. Minayo MCS. The inclusion of violence in the health agenda: historical trajectory. Cien Saude Colet 2006; 11(2):375-383.

2. Krug EG, Mercy JA, Dahlberg LL, Zwi AB, editors. World report on violence and health. In: Krug EG, Mercy JA, Dahlberg LL, Zwi AB, editors. Prefácio. Geneva: World Health Organization; 2002. [documento da Internet]. [acessado 2012 jun. 27]. Disponível em: http://www.who.int/violence_injury_ prevention/violence/world_report/en/index.html.

3. Yunes J. Mortality from Violent Causes in the Americas. Bulletin of PAHO 1993; 27(2):154-167.

4. Yunes J, Zubarew T. Mortalidad por causas violentas en adolescentes y jóvenes: un desafio para la Región de las Américas. Rev. Bras. Epidemiol. 1999; 2(3):102-171.

5. Reichenheim ME, de Souza ER, Moraes CL, Mello Jorge MH, Silva CM, Souza Minayo MC. Violence and injuries in Brazil: the effect, progress made, and challenges ahead. Lancet 2011; 377(9781):1962-1975.

6. Moreno C, Cendales R. Mortalidad y años potenciales de vida perdidos por homicidios en Colombia, 1985-2006. Rev Panam Salud Publica 2011; 30(4): 342-353.

7. López MV, Híjar Medina MC, Rascón Pacheco A, Blanco Muñoz J. Mortality by homicide, the fatal consequences of violence. The case of Mexico, 19791992. Rev Saude Publica 1996; 30(1):46-52.

8. Organização Pan Americana da Saúde. Observatório Regional de Saúde. Leading cause of death. [documento da Internet]. [acessado 2012 jul. 11]. Disponível em: http://new.paho.org/hq/index.php? option $=$ com content $\&$ task=view\&id $=3501 \&$ Itemid $=2391$

9. Organización Panamericana de la Salud. Clasificación estadística internacional de enfermedades y problemas relacionados con la salud. $10^{\text {a }}$ revisión. V.3 Washington D.C: OPS; 1995. (Publicación Científica, n. 554)

10. United Nations Population Division. World Urbanization Prospects, the 2010 Revision, New York: INPD; 2011. [documento da Internet]. [acessado 2012 set 05]. Disponível em: http://esa.un.org/unpd/ wpp/index.htm.

11. Ahmad OB, Boschi-Pinto C, Lopez AD, Inoue M. Age standardization of rates: a new who standard GPE. Discussion Paper Series: n. 31. Geneva: World Health Organization; 2001. [documento da Internet]. [acessado 2012 jun 26]. Disponível em: http:/ /www.who.int/healthinfo/paper31.pdf.

12. SAS 9.3 Software [programa de computador]. Canadá: SAS Institut Inc; 2012. [acessado 2012 jun 26]. Disponível em: http://www.sas.com/software/sas9/

13. Pan American Health Organization. Health Information and Analysis Project. Health Situation in the Americas: Basic Indicators 2012. Washington, DC: United States of America; 2012. [documento da Internet]. [acessado 2012 set 20]. Disponível em: http://ais.paho.org/chi/brochures/2012/BI_ 2012_ENG.pdf.

14. Cerdá M, Morenoff JD, Hansen BB, Tessari Hicks KJ, Duque LF, Restrepo A, Diez-Roux AV. Reducing violence by transforming neighborhoods: a natural experiment in Medellín, Colombia. Am J Epidemiol. 2012; 175(10):1045-1053. 
15. Gawryszewski VP, Monteiro RA, Sá NBS, Mascarenhas MDM, Silva MMA, Bernal R, Malta DC. Acidentes e violências no Brasil: um panorama atual das mortes, internações hospitalares e atendimentos em serviços de emergência. In: Ministério da Saúde. Saúde Brasil 2009: uma análise da situação de saúde e da agenda de nacional e internacional de prioridades em saúde. Brasília: Ministério da Saúde. Secretaria de Vigilância em Saúde. Departamento de Análise de Situação de Saúde; 2010.p.139-173. [documento da Internet]. [acessado 2012 jul. 2]. Disponí vel em: http://portal.saude.gov.br/portal/arquivos/ pdf/29_11_10_saude_brasil_web.pdf.

16. Peres MFT, Almeida JF, Vicentin D, Cerda M, Cardia N, Adorno S. Queda dos homicídios no município de São Paulo: uma análise exploratória de possíveis condicionantes. Rev. Bras. Epidemiol. 2011; 14(4):709-721.

17. Chauhan P, Cerdá M, Messner SF, Tracy M, Tardiff K, Galea S. Race/Ethnic-Specific Homicide Rates in New York City: Evaluating the Impact of Broken Windows Policing and Crack Cocaine Markets. Homicide Stud. 2011; 15(3):268-290.

18. Hernández-Bringas H, Narro-Robles J. El homicidio en México, 2000-2008. Pap. Poblac 2010; 16(63): 243-271.

19. Gutiérrez-Trujillo G, Fernández-Cantón SB and Viguri-Uribe R. Homicidios en menores de 15 años de edad, México 2000-2010. Informe preliminar. Bol. Med. Hosp. Infant. Mex. 2011; 68(3):253-258.

20. Romero-Salazar A, Rujano R, Raima RR, Sulbarán MA. Agresividad cotidiana y aprobación de la violencia extrema. Estud. Soc 2009; 17(33):259-280.

21. Vargas Sanabria M, Solano Calderón L \& Bonilla Montero R. Análisis Médico Legal de los homicidios en Costa Rica en el 2008. Med. Leg 2009;26(2):7-27.

22. Lemard G, Hemenway D. Violence in Jamaica: an analysis of homicides 1998-2002. Inj Prev. 2006; 2(1):15-18.

23. González-Pérez GJ, Vega-López MG, Vega-López A, Muñoz-De-La-Torre A, Cabrera-Pivaral CE. Homicidios de adolescentes en México, 1979-2005: evolución y variaciones sociogeográficas. Pap. Poblac 2009; 15(62):109-141.

24. Informe Mundial sobre las drogas 2010. Oficina de las Naciones Unidas Contra la Droga y el Delito. Nova York: Naciones Unidas; 2010.

25. Franco S. A social medical approach. Am J Public Health 2003; 93(12):2032-2036.

26. Ministério da Saúde. Secretaria de Vigilância em Saúde. Análise ecológica da violência letal no município de Vitória-ES: sua distribuição e contexto socioespacial. In: Ministério da Saúde. Saúde Brasil 2007: uma análise da situação de saúde. Brasília: Ministério da Saúde; 2008. p. 457-508. [documento da Internet]. [acessado 2012 jun 27]. Disponível em: http://portal.saude.gov.br/portal/arquivos/pdf/saude_brasil_2007.pdf.

Artigo apresentado em 10/05/2012

Aprovado em 23/07/2012

Versão final apresentada em 13/09/2012 